\title{
UPDATES IN THE MANAGEMENT OF NEUROBLASTOMA
}

\author{
Roly Squire \\ Department of Surgery, Leeds General Infirmary, Leeds, United Kingdom \\ Received: 30 August 2016 / Accepted: 31 August 2016
}

\begin{abstract}
Through international collaboration, we are at the start of a new age in the management of this enigmatic tumour. Tumours are now grouped at diagnosis using the international neuroblastoma risk grouping (INRG), which uses information from tumour tissue reflecting tumour biology, as well as radiology to define image-defined risk factors. Tumours in high-risk groups receive maximal therapy in an attempt to try and improve outcomes which are still poor. Intermediate-risk tumours, which have better outcomes due to better response to the current therapy, are treated aggressively with combination therapies with proven therapeutic effects but with increasing attention to the minimising adverse treatment effects. The treatment of low-risk tumours is now vastly reduced, acknowledging the excellent outcome in these children using minimal therapy. It has become apparent that the consequences of therapy in these children can easily be worse than those from the tumour itself. For very low-risk tumours, trials are exploring the outcomes without any treatment. INRG allows more precise comparison of results between different international groups and provides a template into which new prognostic variables can be introduced, and their value assessed. In many centres, multiarray analysis is studying the genetic profile of each tumour; perhaps, this will allow increasing individualisation of treatment programmes in the near future.
\end{abstract}

Key words: Image-defined risk factors, international neuroblastoma risk grouping, neuroblastoma

\section{Introduction}

The enigma of neuroblastoma may be close to being solved: The spectrum of tumours from benign in some infants to one of the most aggressive solid tumours in the toddler. These are exciting times. As a result of stratification of risk at diagnosis, we are now starting to personalise therapy, sometimes escalating chemotherapy and introducing new combination therapies and sometimes reducing the therapeutic burden yet still achieving good outcomes.

Neuroblastoma is the most common extracranial solid tumour of childhood in Europe and North America, although the incidence is probably lower in much of Asia. Of all paediatric tumours, neuroblastoma has become the target for the most innovative approaches, either completely novel or by the introduction of adult agents in the younger age, Yet, high-risk neuroblastoma remains one of the hardest paediatric tumours to cure.The current therapies include

Correspondence: Roly Squire, Consultant Paediatric Surgeon, Leeds General Infirmary, Leeds LS1 3EX, United Kingdom.

Email: r.squire@nhs.net aggressive surgical resection; chemotherapy intensification using myeloablative chemotherapy with stem cell rescue; radiotherapy to consolidate local control; immunotherapy and differentiation therapy. Isotope therapy is being explored as part of standard induction therapy, therapy for tumours resistant to chemotherapy and for relapse. At the other extreme, for low-risk tumours, only minimal therapy is required, and an increasing number of tumours are now being observed, without any interventions.

This review will focus primarily on the introduction of the new approach to risk grouping, an international guide to the selection of treatment for individual children. This is the template on which specific treatment modalities are superimposed and allows the clinician to seek out the latest chemotherapy, surgical or new agent advice appropriate for the child, he or she is treating.

\section{Presentation and Initial Investigations}

There are three main modes of presentation of neuroblastoma, largely a consequence of stage and site of the tumour: 
- Symptoms from metastatic spread

- Symptoms from the primary tumour

- Incidental finding of primary tumour

In a toddler, it is commonly the spread to bones that are first observed; for instance, a child who develops a limp or stops walking. Another typical presentation is bilateral periorbital swelling and bruising ('raccoon eyes') caused by soft tissue metastases. A notable feature of children who present with these patterns of metastatic disease is that they appear to be generally unwell, a consequence of associated bone marrow spread. Imaging of the affected part of the child's body will often give an indication of the malignant cause, and an abdominal ultrasound and chest radiograph should be early investigations of any young child who is generally unwell, off food, with a haematological picture which includes anaemia but has ruled out leukaemia.

An infant with metastatic disease (details of MS/4S disease are described later) more commonly present with hepatomegaly which can be so gross that ventilation is compromised, and/or soft tissue lumps in the skin. Usual features of metastatic neuroblastoma in infancy are that the primary tumour is quite small and the bone marrow involvement light.

A large primary tumour may cause symptoms at any site; respiratory compromises if in the chest and abdominal pain in the abdomen, but the most concerning local event come from a paravertebral primary tumour which extends into the spinal canal. It is of concern that symptomatic spinal cord compression in younger children often presents too late for intervention to prevent permanent damage. Neuroblastoma should always be considered when a child who can walk goes of his or her legs without any other more obvious explanation. Urgent spinal imaging will demonstrate the tumour, and emergency treatment is indicated while the results of diagnostic investigations are awaited.

Most neuroblastomas can be diagnosed by the presence of raised urinary catecholamine metabolites (vanillylmandelic acid - VMA; and homovanillic acid - HMA). Urinary dopamine is also frequently elevated. Unlike for pheochromocytomas, a spot urinary analysis is adequate for diagnosis of neuroblastoma, not requiring a $24 \mathrm{~h}$ collection, but clinicians should be aware that raised catecholamines in neuroblastoma can have important clinical implications, causing hypertension at presentation and risks of circulatory disturbance during anaesthetic procedures, especially when the tumour is handled.

Asymptomatic tumours may be discovered incidentally. This is a common presentation of a non-metastatic thoracic primary tumour, but also adrenal tumours that are present at birth and can be seen on an ante-natal ultrasound. The general principles of management are the same for incidental primary tumours as for those who have symptoms, but antenatal adrenal tumours and those presenting in the neonatal period do appear to be a separate group due to their exceptionally good prognosis, and so these are considered in a separate section of this review.

For all other suspected neuroblastomas (i.e. all tumours presenting outside of the neonatal period), a tissue diagnosis is now mandatory, even if the VMA and

HVA are elevated. This is because histological and biological information only obtainable from tumour tissue is essential to guide treatment. Initial imaging is carried out to decide whether a tissue biopsy should be carried out (metastatic or unresectable primary tumours) or attempted resection (localised and resectable primary tumours).

To identify metastatic spread, the current guidelines recommend chest computed tomography (CT) scan, bone marrow aspiration and trephine and metaiodobenzylguanidine (MIBG) scan. Bone scan may also be considered but is unlikely to add value to the results from the MIBG scan. Recommended imaging for the primary tumour is a magnetic resonance scan since this generally provides superior definition of the involvement of adjacent organs than CT. However, CT and ultrasound in combination will often provide enough information for all but the hardest cases.

Finally, there is an unusual paraneoplastic presentation of some very small primary neuroblastomas: Opsoclonus/ myoclonus (OM). A child presenting with $\mathrm{OM}$ will generally be referred for a neurological opinion, and investigation for an underlying neuroblastoma with urinary catecholamine assay and appropriate imaging should always be carried out. Many neurologists believe that even those in whom no neuroblastoma is identified 
Table 1: INSS (including 1993 modifications) ${ }^{[1]}$

\begin{tabular}{l|l}
\hline Stage 1 & $\begin{array}{l}\text { Localised tumour with complete gross excision, with or without microscopic residual disease: Representative } \\
\text { ipsilateral lymph nodes negative for tumour microscopically: Nodes attached and removed with tumour may be } \\
\text { positive }\end{array}$ \\
\hline Stage 2a & $\begin{array}{l}\text { Localised tumour with incomplete gross excision: Representative ipsilateral non-adherent lymph nodes negative } \\
\text { for tumour microscopically }\end{array}$ \\
\hline Stage 2b & $\begin{array}{l}\text { Localised tumour with or without complete gross excision, with ipsilateral non-adherent lymph nodes positive: } \\
\text { Enlarged contralateral lymph nodes negative microscopically }\end{array}$ \\
\hline Stage 3 & $\begin{array}{l}\text { Unresectable unilateral tumour infiltrating across the midline (beyond the opposite side of the vertebral col- } \\
\text { umn) with or without regional lymph node involvement: Or midline tumour with bilateral extension through } \\
\text { infiltration (unresectable) or lymph node involvement }\end{array}$ \\
\hline Stage 4 & $\begin{array}{l}\text { Any primary tumour with dissemination to distant lymph nodes, bone, bone marrow, liver, skin and/or other } \\
\text { organs (except as defined for Stage 4s disease) }\end{array}$ \\
\hline Stage 4s & $\begin{array}{l}\text { Localised primary tumour (as defined for Stage 1, 2a or 2b disease) with dissemination limited to skin, liver and/ } \\
\text { or bone marrow, limited to infants younger than 1 year (marrow involvement of<10\% of total nucleated cells } \\
\text { and MIBG scan findings negative in the tumour) }\end{array}$ \\
\hline
\end{tabular}

INSS: International neuroblastoma staging system, MIBG: Metaiodobenzylguanidine

Table 2: Definition of risk groups (patients with 5-year event-free survival)

\begin{tabular}{l|c}
\hline Very low risk (\%) & $>85$ \\
\hline Low risk (\%) & $77-85$ \\
\hline Intermediate risk (\%) & $50-75$ \\
\hline High risk (\%) & $<50$ \\
\hline
\end{tabular}

have occult neuroblasts underlying this condition, and the non-surgical management of those with or without an identified tumour is much the same. Trials are currently trying to establish the best treatment for this distressing condition. Resection of the primary tumour can occasionally be effective for symptom improvement, and so should be carried out, even though the tumour in this scenario does not behave in a malignant fashion.

\section{Staging Systems and Risk Grouping}

In 1971, Audrey Evans published the original staging system for children with neuroblastoma, critically recognising that neuroblastoma was unique in having a group of patients, the infants, who could have a metastatic tumour, yet with a better outcome than some with localised disease. She named this stage IVS, the 'S' standing for special, and this subgroup has remained special.

The unexpectedly good outcomes in infant neuroblastoma make it the most common reason for spontaneous tumour regression in humans. The other stages more traditionally include I-III (1-3) for localised tumours and IV (4) for classical metastatic disease, and overtime these have been modified and agreed by international committees, resulting in the international neuroblastoma staging system (INSS) in 1986 [Table 1]. ${ }^{[1]}$

INSS remains the current surgical staging system, but over the years, there has been increasing awareness that surgical staging for neuroblastoma is flawed. First, patient outcomes are strongly influenced by the biological activity of the tumour, which can be extremely variable, Evans Stage $4 \mathrm{~s}$ tumours being a prime example. A predictive classification was required to include measurement of biological markers. Second, it became apparent that the surgical approach could influence the surgical staging: The same large tumour crossing the midline could be Stage 1 if resected completely, Stage 2 if incompletely resected or Stage 3 if just biopsied. How can one compare the impact of other treatments in the context of such inconsistency? Furthermore, case reports of infant tumours managed without surgery were accumulating, and these need to be included in staging systems to avoid exclusion bias.

In 2005, an international collaboration of neuroblastoma specialists met to address this issue and set up a project to combine datasets from all of the available national and international tumour registries, using survival tree 
Table 3: Image-defined risk factors ${ }^{[4]}$

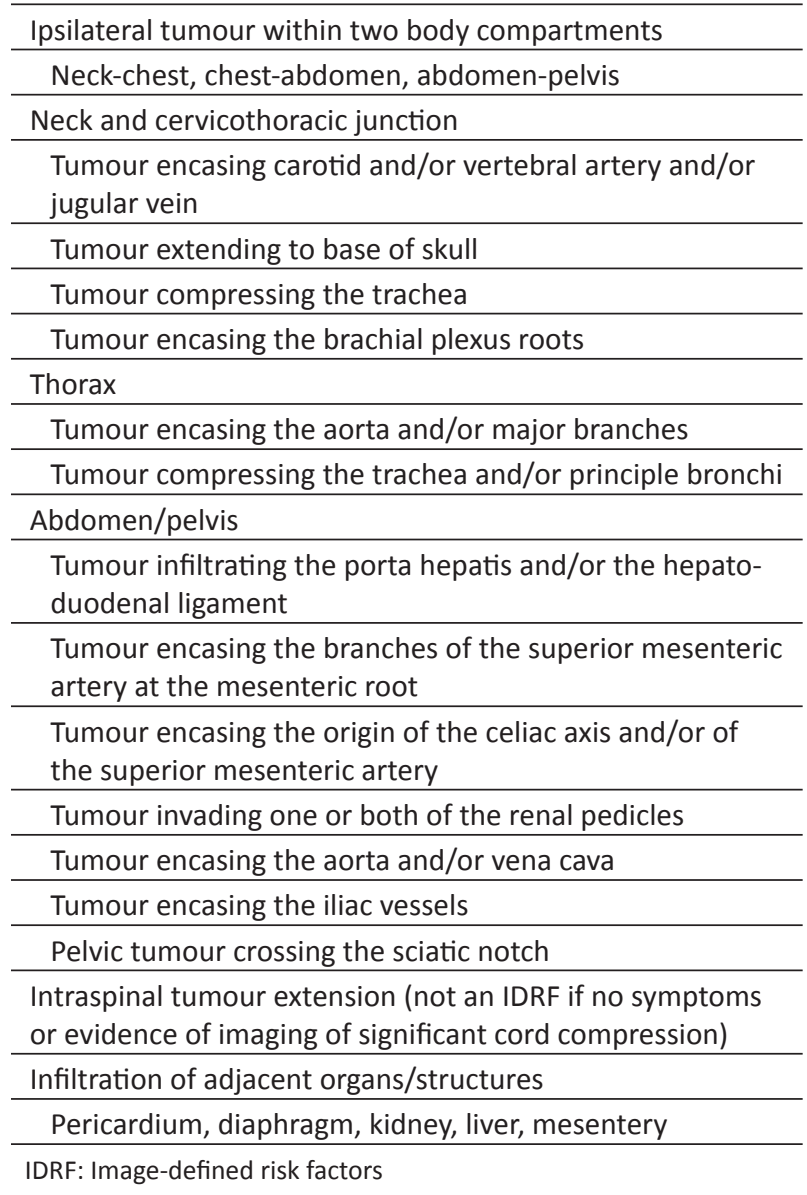

Table 4: INRG staging system ${ }^{[4]}$

\begin{tabular}{l|l}
\hline L1 & $\begin{array}{l}\text { Localised tumour not involving vital structures as } \\
\text { defined by the list of image-defined risk factors and } \\
\text { confined to one body compartment }\end{array}$ \\
\hline L2 & $\begin{array}{l}\text { Locoregional tumour with presence of one or more } \\
\text { image defined risk factors }\end{array}$ \\
\hline M & Distant metastatic disease (except MS) \\
\hline MS & $\begin{array}{l}\text { Metastatic disease in child under 18 months, with } \\
\text { metastases confined to skin, liver and/or bone marrow }\end{array}$ \\
\hline
\end{tabular}

INRG: International neuroblastoma risk grouping

regression analysis of data collected on 8800 patients to identify the most important factors at diagnosis which impact on outcome. ${ }^{[2]}$ Using these indicators of outcome, it was possible to place children with neuroblastoma into risk groups at diagnosis [Table 2]: High, intermediate, low and very low.
The analysis showed seven parameters which appear to have the greatest independent impact on the patient outcome, and as a consequence need to be scored to decide which risk group a child is in at presentation is as follows:

- Age

- Stage

- Histological category

- Grade of tumour differentiation

- MYCN amplification

- Segmental chromosome abnormalities (LOH 11q)

- DNA ploidy.

A critical component of risk grouping at diagnosis was the removal of surgical staging as the method of classifying the primary tumour. Previous work on the relation between radiological definition of potential surgical risk factors and surgical complications in localised tumours ${ }^{[3]}$ was extended to the assessment of all tumours. It should be recognised that this was a speculative step, intended primarily to allow comparison of data between studies. The presence of radiological risk factors, now referred to as image-defined risk factors (IDRF), does not necessarily equate to resectability. However, it can reasonably be assumed that a tumour with IDRFs will be harder to resect, and so surgery is likely to carry more risk. This should be taken into account when assessing the balance of risk versus benefit of resection, and increasingly, guidelines have suggested that if there are IDRFs at presentation, the tumour should be biopsied and resection only attempted after chemotherapy. A list of the IDRF is in Table 3.

Additional to the development of risk groups, the international neuroblastoma risk grouping (INRG) task force has achieved consensus guidance on standardisation of histological classification, grading of differentiation and genetic evaluation. This work continues, and it can be expected that there will be modifications to the definitions as more data and outcomes are analysed.

The use of IDRFs at diagnosis is incompatible with the use of INSS as the staging within INRG, and so the task force introduced a new international neuroblastoma risk group staging system ${ }^{[4]}$ which is listed in Table 4.

Finally a complex table can be constructed to use an individual patient's score from the seven prognostic variables to derive an estimation of which risk group the 
Table 5: INRG consensus pre-treatment classification schema ${ }^{[2]}$

\begin{tabular}{l|l|l|l|l|l|l|l}
\hline $\begin{array}{l}\text { INRG } \\
\text { stage }\end{array}$ & $\begin{array}{c}\text { Age } \\
\text { (months) }\end{array}$ & $\begin{array}{l}\text { Histologic } \\
\text { category }\end{array}$ & $\begin{array}{l}\text { Grade of tumour } \\
\text { differentiation }\end{array}$ & MYCN & $\begin{array}{l}\mathbf{1 1 q} \\
\text { LOH }\end{array}$ & Ploidy & $\begin{array}{l}\text { Pre-treatment } \\
\text { risk group }\end{array}$ \\
\hline L1/L2 & & GN maturing & & & & & A: Very low \\
\hline L1 & & GNB intermixed & & NA & & & \\
\hline & & $\begin{array}{l}\text { GN maturing or } \\
\text { GNB intermixed }\end{array}$ & & Amp & & & K: High \\
\hline L2 & $<18$ & $\begin{array}{l}\text { Any, except GN } \\
\text { maturing or GNB } \\
\text { intermixed }\end{array}$ & & NA & No & & D: Low \\
\hline & $18+$ & $\begin{array}{l}\text { GNB nodular } \\
\text { neuroblastoma }\end{array}$ & Differentiating & NA & No & & G: Intermediate \\
\hline & & & $\begin{array}{l}\text { Poorly differenti- } \\
\text { ated }\end{array}$ & NA & Yes & & H: Intermediate \\
\hline & & & Amp & & & N: High \\
\hline M & $<18$ & & & NA & & Hyperdiploid & F: Low \\
\hline & $<12$ & & & NA & & Diploid & I: Intermediate \\
\hline & $12-<18$ & & & NA & & Diploid & J: Intermediate \\
\hline & $<18$ & & & NA & & & O: High \\
\hline & $18+$ & & & NA & No & & O: High \\
\hline MS & $<18$ & & & Amp & & & C: Very low \\
\hline & & & & Yes & & R: High \\
\hline & & & & &
\end{tabular}

GN: Ganglioneuroma, GNB: Ganglioneuroblastoma, Amp + amplified, NA: Not amplified

child falls into [Table 5]. Although the table appears to be complex, it becomes simple to use when managing a single patient.

\section{High-risk Disease}

As can be seen from the table, high-risk neuroblastoma is predominantly defined by the same characteristics as before the introduction of INRG: Stage 4 disease outside of infancy. However, the poor prognostic impact of MYCN amplification also features strongly in all, but the best prognosis localised tumours. High-risk treatment protocols vary in detail with regard to induction chemotherapy, timing of surgery and dose/extent of radiotherapy, but the treatment principles are the same for all of the major paediatric cancer collaborative groups. High-intensity induction chemotherapy is followed by the assessment of response. Failure of response to induction chemotherapy results in immediate introduction of the second-line chemotherapy. If the response is adequate at this and subsequent assessments generally measured by resolution of metastatic disease, resection of the primary tumour is planned. If the surgeon considers that resection may carry a high morbidity at this time, there is the option to delay surgery until later in the protocol. An example might be if there is a particular risk of renal insufficiency that might delay treatment intensification since this delay confers a worse overall outcome.

The surgery should attempt complete resection even if the tumour is still invasive and encasing major vessels such as the aorta. Early results from the high-risk study from the International Society of Paediatric Oncology suggest a better overall outcome if the resection is complete or near complete.

Before the resection stem cells are harvested, and following surgical recovery, the child undergoes 
intensification with myeloablative chemotherapy and stem cell rescue. Following this, there is radiotherapy, which may be intensified in areas where there is evidence of residual tumour from the tumour histology. The last therapeutic intervention is immunotherapy, using the anti-GD2 monoclonal antibody, with or without IL-2. All of these interventions are highly toxic and associated with significant morbidity. Once treatment is complete and assuming that the child appears to be in remission, maintenance differentiation therapy using 13 -cis-retinoic acid is given since this has been shown to delay relapse in those who do subsequently develop recurrent disease.

The treatment burden for high-risk disease is very high, but this intensive regime has been shown to slowly improve the outcome in this terrible tumour, with 5-year survival steadily approaching $50 \%$.

\section{Low- and Intermediate-risk Disease}

These tumours will be expected to show a better response to chemotherapy, and it is for these children that the stratification of treatment is so important. Historically, many children with low-risk disease were overtreated, and some will have had a worse outcome as a result of treatment than they would have if they had received no treatment at all. Localised tumours without IDRF will need resection at diagnosis, and some will need no further treatment. Tumours with IDRF at presentation, and infants with metastatic disease but no MYCN amplification, will need biopsy and chemotherapy. Some of these will continue to have IDRF even after chemotherapy, and in many of these, an observational policy is being trialled. However, IDRF has not been validated in this role, and it is for the clinical team to decide in individual cases whether the risks from surgery outweigh the risks from recurrent disease. Advice may be sought from national and international coordinators.

Surprisingly, MS disease can present a particularly tricky problem. In principle, treatment can be avoided, with the expectation that the primary tumour and metastases will spontaneously resolve. However, the gross liver disease can present the child with too great a tumour burden, and urgent intervention may be required. In this circumstance, the infant may be unable to tolerate even reduced or minimal doses of chemotherapy, and so this self-limiting disease can still be fatal. Application of an abdominal silo is sometimes required to manage abdominal compartment syndrome and renal failure.

\section{Recurrent Disease}

It is rare for there to be long-term survival following treatment of recurrent neuroblastoma. However, this is a fertile area for the introduction of novel strategies, and there are numerous new therapies which are being trialled in these children, hoping for a response that will introduce a new agent to our therapeutic array. An exciting area of development is with radioisotope therapy. MIBG has been used in a number of trials with some success, although the optimum use of MIBG is still being clarified. Other agents such as lutetium dotatate are being introduced with some optimism since they appear to target even drug-resistant neuroblasts.

\section{Perinatal Adrenal Masses}

One group of children who should be managed differently is those under 44 weeks gestational age at the time of diagnosis, who have a small $(<5 \mathrm{~cm})$ adrenal mass. This group may include some infants with other diagnoses such as neonatal adrenal haemorrhage, but irrespective of the baseline urinary catecholamine, there is now good evidence that it is safe to manage the whole group expectantly, avoiding any interventions and minimising investigations until the child is older. The tumour and catecholamines must be monitored since the rare tumour which is more aggressive needs to be identified, and treatment started. For the rest, an MIBG scan at age 3 months will rule out metastatic disease, and if the tumour shrinks, then it will not need to be removed.

\section{Conclusion}

While treatment intensification is still being pursued in high-risk neuroblastoma, there are many tumours which respond to quite a low therapeutic burden, and in some, no treatment is required. The international collaboration pioneered by the INRG task force allows us to standardise pre-treatment classification of tumours and compare like with like when results from different groups are published. We expect this to speed up progress in treating neuroblastomas of all risk groups. 


\section{Conflict of Interest}

The authors declare that they have no conflict of interest.

\section{References}

1. Brodeur GM, Pritchard J, Berthold F, et al. Review of the international criteria for neuroblastoma diagnosis, staging and response to treatment. J Clin Oncol 1993;11:1466-77.

2. Cohn SL, Pearson AD, London WB, et al. The international neuroblastoma risk group (INRG) classification: An INRG task force report. J Clin Oncol 2009;27:289-97.

3. Cecchetto G, Masseri V, De Bernardi B, et al. Surgical risk factors in primary surgery for localised neuroblastoma: The LNESG1 study of the European international society of pediatric oncology neuroblastoma group. J Clin Oncol 2005,23:8483-9.

4. Monclair T, Brodeur GM, Ambros P, et al. The international neuroblastoma risk group (INRG) staging system: An INRG task force report. J Clin Oncol 2009;27:298-303. 